\title{
A REGULAR QUATERNION POLYGON
}

\author{
Donald W. Crowe
}

(received February 9, 1959)

Repeated application of the unitary reflections

$$
R=\frac{1+i}{2}\left(\begin{array}{rr}
1 & -1 \\
1 & 1
\end{array}\right) \text { and } S=\left(\begin{array}{ll}
1 & 0 \\
0 & i
\end{array}\right)
$$

to the point $(1,0)$ and line $x+i y=1$ yields 24 points and 24 lines. These are the vertices and edges of the regular complex polygon $4\{3\} 4$ whose group has the abstract definition $R^{4}=I, R S R=$ SRS [1]. The purpose of this note is to introduce the notion of regular quaternion polygon and give an example analogous to the preceding one.

We consider a two-dimensional "quaternion unitary" space, $Q_{2}$. This is the space whose points are pairs $(x, y)$ of quaternions, with the distance between two points $\left(x_{1}, y_{1}\right)$ and $\left(x_{2}, y_{2}\right)$ defined by

$$
\left[\left(x_{1}-x_{2}\right)\left(\overline{x_{1}-x_{2}}\right)+\left(y_{1}-y_{2}\right)\left(\overline{y_{1}-y_{2}}\right]^{\frac{1}{2}}\right. \text {. }
$$

A quaternion unitary (or simply unitary) transformation is one which preserves distance. A line is the set of points satisfying some quaternion equation $a x+b y=c$. A reflection is a unitary transformation of finite period $(>1)$ which leaves a line pointwise fixed. A regular quaternion polygon is a configuration of points and lines ("vertices" and "edges") in $Q_{2}$ which is transformed into itself by a group generated by two unitary reflections, one which cyclically permutes the vertices on one edge and another which cyclically permutes the edges through one of these vertices.

The following example of a polygon with 80 vertices and 80 edges is an obvious analogue of the $4\{3\} 4$ described above.

Can. Math. Bull. Vo1. 2, No. 2, May 1959 
The unitary reflection

$$
R=\frac{1+i}{2}\left(\begin{array}{rr}
1 & -1 \\
1 & 1
\end{array}\right)
$$

cyclically permutes the points

$(1,0),([1+i] / 2,[-1-i] / 2),(0,-i)$, and $([1-i] / 2,[1-i] / 2)$ on the line $x+i y=1$. The unitary reflection

$$
T=\left(\begin{array}{ll}
1 & 0 \\
0 & j
\end{array}\right)
$$

permutes cyclically the lines $x+i y=1, x+k y=1, x-i y=1$, and $x-k y=1$ each containing the point $(1,0)$. Let the four quaternions $\pm \mathrm{a}, \pm \mathrm{aj}$ be denoted by $4^{\mathrm{a}}$. Then repeated application of $R$ and $T$ to $(1,0)$ yields the 80 points

$$
\begin{aligned}
& \left({ }_{4} 1,0\right),\left(0,{ }_{4} 1\right),\left(4^{i}, 0\right),\left(0,4^{i}\right), \\
& \left({ }_{4}[1+i] / 2,4[1+i] / 2\right),\left({ }_{4}[1-i] / 2,4[1-i] / 2\right), \\
& \left({ }_{4}[1+k] / 2,4[1-k] / 2\right),\left({ }_{4}[1-k] / 2,4[1+k] / 2\right) .
\end{aligned}
$$

These lie by fours on the 80 images of the line $x+i y=1$. Together these are the vertices and edges of our regular quaternion polygon.

The group $\{R, T\}$ generated by $R$ and $T$ has at least 320 elements, for any one of the 320 figures consisting of an edge and a vertex on it can be transformed into any other by an element of the group. But the group also has at most 320 elements, for $R$ and $T$ satisfy the relations

$$
R^{4}=I, \quad R T R T R=T R T R T,\left(R T^{2}\right)^{2}=\left(T^{2} R\right)^{2},(R T)^{5}=\left(R T^{2}\right)^{4} .
$$

Any group whose generators satisfy these relations can be shown by enumeration of cosets [2; pp. 12-17] to have order at most 320. Consequently these relations constitute an abstract definition of the group $\{R, T\}$.

The 80 vertices of the polygon lie by eights on the $10 \mathrm{dia}-$ gonals $x=0, y=0, y=4 x$, and $y=4$ ix. The "slopes" of these diagonals are $\infty, 0,4^{1}, 4^{i}$. These slopes correspond to points $\infty,(0,0,0,0),( \pm 1,0,0,0),(0, \pm 1,0,0),(0,0, \pm 1,0)$, and $(0,0,0, \pm 1)$ of a four dimensional Euclidean space $\mathrm{E}_{4}$. More conveniently, let this $E_{4}$ be the equatorial hyperplane of a unit sphere in $E_{5}$, and project the 10 points stereographically onto this sphere. 
The only points which are moved by this projection are $\infty$ and $(0,0,0,0)$; the 10 points on the sphere are readily seen to be the vertices of the five dimensional cross polytope, $\beta_{5}$. Furthermore, each of the diagonals can itself be considered to be an $E_{4}$. The eight points of the polygon lying in a diagonal are then the eight vertices of a $\beta_{4}$.

\section{REFERENCES}

1. H.S.M. Coxeter, The groups of the regular polygons, Can. Math. Congress Newsletter, Sept. 1956.

2. H.S.M. Coxeter and W.O.J. Moser, Generators and Relations for Discrete Groups, Ergeb. d. Math. 14, (Berlin, 1957).

University of Toronto 